\title{
Prevalence of multimorbid degenerative lumbar spinal stenosis with knee and/or hip osteoarthritis: protocol for a systematic review and meta-analysis
}

James J. Young ${ }^{1,2^{*}}$ (D) Jan Hartvigsen ${ }^{1,3}$, Rikke K. Jensen ${ }^{1,3}$, Ewa M. Roos ${ }^{1}$, Carlo Ammendolia ${ }^{4,5}$ and Carsten Bogh Juhl,

\begin{abstract}
Background: Lumbar spinal stenosis (LSS) and knee and hip osteoarthritis (OA) are prevalent conditions in the aging population and published literature suggests they share many symptoms and often are present at the same time in patients. However, no prevalence estimates of multimorbid LSS and knee and/or hip OA are currently available. The primary objective of this systematic review is therefore to estimate the prevalence of multimorbid LSS with knee and/or hip OA using radiological, clinical, and combined case definitions.

Methods: This systematic review protocol has been designed according to the guidelines from the Cochrane Collaboration and is reported according to the Preferred Reporting Items for Systematic Reviews and Meta-Analysis Protocols. A comprehensive search will be performed in the following databases: MEDLINE, EMBASE, CENTRAL, and CINA $\mathrm{HL}$. Forward citation tracking will be performed in Web of Science. No restriction for publication date and language will be applied in the literature search, but only articles in English will be included. The search strategy will include the following domains: LSS, knee OA, and hip OA. Retrieved citations will be screened by two authors independently. Disagreements will be discussed until consensus, and a third reviewer will be consulted if consensus cannot be reached. Data extraction and assessment of risk of bias assessment will be done by two authors independently, using a standardized data extraction form and a modified risk of bias tool for prevalence studies. Meta-analysis estimating prevalence with $95 \% \mathrm{Cl}$ will be performed using a random effects model. Meta-regression analyses will be performed to investigate the impact of the following covariates: LSS clinical presentations, sample population, healthcare setting, risk of bias, and other patient characteristics on prevalence estimates for multimorbid LSS and knee and/or hip OA.
\end{abstract}

Discussion: The results of this review will provide the first estimates of the prevalence of multimorbid LSS and hip and knee OA based on various case definitions. The impact of covariates such as LSS clinical presentations, sample population, healthcare setting, risk of bias, and patient characteristics on prevalence estimates will also be presented.

(Continued on next page)

\footnotetext{
* Correspondence: jyoung@health.sdu.dk

${ }^{1}$ Center for Muscle and Joint Health, University of Southern Denmark, Campusvej 55, 5230 Odense M, Denmark

${ }^{2}$ Research Division, Canadian Memorial Chiropractic College, 6100 Leslie Street, Toronto, Canada

Full list of author information is available at the end of the article
}

(C) The Author(s). 2020 Open Access This article is licensed under a Creative Commons Attribution 4.0 International License, which permits use, sharing, adaptation, distribution and reproduction in any medium or format, as long as you give appropriate credit to the original author(s) and the source, provide a link to the Creative Commons licence, and indicate if changes were made. The images or other third party material in this article are included in the article's Creative Commons licence, unless indicated otherwise in a credit line to the material. If material is not included in the article's Creative Commons licence and your intended use is not permitted by statutory regulation or exceeds the permitted use, you will need to obtain permission directly from the copyright holder. To view a copy of this licence, visit http://creativecommons.org/licenses/by/4.0/ The Creative Commons Public Domain Dedication waiver (http://creativecommons.org/publicdomain/zero/1.0/) applies to the data made available in this article, unless otherwise stated in a credit line to the data. 
(Continued from previous page)

Systematic review registration: PROSPERO, awaiting registration

Keywords: Lumbar spinal stenosis, Hip osteoarthritis, Knee osteoarthritis, Multimorbidity, Prevalence, Systematic review protocol

\section{Background}

Musculoskeletal health is considered an important prerequisite for healthy aging [1]. Unfortunately, musculoskeletal pain is a leading and growing cause of disability $[2,3]$. Low back pain and osteoarthritis (OA) are among the most disabling chronic conditions globally, ranking as the first and twelfth causes of years lived with disability, respectively [4]. Chronic conditions are also the leading cause of the increased prevalence of multimorbidity (presence of two or more co-occurring diseases) among older individuals (estimated $67 \%$ of Americans over the age of 65 ) and individuals experience increased functional limitations with each additional chronic disease [5]. However, the impact of multimorbid musculoskeletal conditions on both patients and healthcare systems has not been extensively studied.

Lumbar spinal stenosis (LSS) is a lumbar spine condition that occurs with increasing age and is associated with substantial pain and disability in older adults [6]. LSS is considered one of the most burdensome spinal conditions [7, 8] and is the leading reason for spinal surgery in the elderly [9]. A recent systematic review found the prevalence of symptomatic LSS to be $11 \%$ in the general population [10], and almost half of those over the age of 60 experience symptomatic LSS [11]. The number of individuals with disability attributed to LSS is expected to rise globally due to the rapidly increasing population over the age of 60 years [12]. Even with most patients experiencing substantial improvement from surgical intervention, pain and disability persist at long-term follow-up [13]. One possible explanation for the continuing symptoms in these patients may be the presence of comorbid musculoskeletal conditions, as comorbid conditions limiting walking ability, including knee and hip arthrosis, predict worse surgical outcomes [14].

Multimorbidity in the aging population is becoming increasingly recognized as an important health determinant [15-17]. Musculoskeletal pain often occurs in more than one body site [18-20]. An Australian study found $61 \%$ of women in the sample had multi-joint pain, with low back pain (35\%) and knee pain (27\% right, 24\% left) as the most common locations, while hip pain was less common (15\% right, $16 \%$ left) [21]. OA has been shown to be associated with other comorbid conditions [22-24], including other musculoskeletal conditions [24]. In one sample of patients with clinically diagnosed knee OA, 55\% reported back pain, and the presence of back pain was associated with worsened levels of pain and disability [25].
LSS can also coexist with other musculoskeletal conditions, including OA $[26,27]$. One study found a mean of two comorbidities (less than $20 \%$ reported no comorbidities) in LSS patients, with lower limb arthrosis included among the most common comorbidities [28]. Hip-spine syndrome has been described in the literature to define coexisting hip and lumbar spine disorders and was originally developed to describe concomitant degenerative spine and hip disease [29]. Particularly interesting is the relation between hip OA and degenerative LSS, as these conditions share a similar degenerative etiology and radiographic findings of degeneration occur in both the lumbar spine and hip [11, 30-32]. Clinical reports have documented patients with co-occurring LSS and hip OA [27, 33], but the relationship with co-occurring knee OA [34], is relatively unknown. However, there is evidence suggesting knee $\mathrm{OA}$ and low back pain commonly co-exist [24] and low back pain has been identified as a risk factor for the development of knee pain in older adults [35]. A recent study also found the presence of radiographic knee OA was associated with all phenotypes of spinal OA (odds ratios ranging from 1.8 to 4.3) [36], which may represent the spinal changes found in LSS.

As the number of individuals with LSS, knee OA, and hip OA rises, it is likely that many older individuals will experience these conditions comorbidly. In fact, the number of individuals living with multimorbidity is increasing as a result of the aging global population [15]. A substantial economic burden has been attributed to multimorbidity in older adults [37] due to functional decline and loss of independence [38]. While it is likely that comorbid LSS and knee and/or hip OA will impact disability levels and healthcare costs, a more developed understanding of the magnitude of this growing health concern is required for informed prioritization and management of these individuals. We are unaware of any formal attempts to estimate the prevalence of multimorbid LSS with knee and/or hip OA. Thus, we do not know how often these conditions co-occur. It is unknown if the relationship between LSS and OA is an incidental imaging finding and unrelated to more severe symptoms and disability or if both conditions have a unique contribution to the health state of patients. As such, multimorbid prevalence estimates using a variety of definitions of LSS and $\mathrm{OA}$ are needed. 


\section{Objectives}

The overall objective of this systematic review is to estimate the prevalence of multimorbid degenerative LSS with knee and hip OA, respectively.

The primary outcome will be the prevalence of degenerative LSS defined by a combination of clinical evaluation and imaging with co-occurring (i) knee OA and (ii) hip OA.

Secondary outcomes will be the prevalence of degenerative LSS defined by clinical evaluation and cooccurring (i) knee OA and (ii) hip OA and the prevalence of degenerative LSS defined by imaging and cooccurring (i) knee OA and (ii) hip OA.

\section{Methods}

This protocol has been prepared according to the guidelines from the Cochrane Collaboration [39] and reported according to the Preferred Reporting Items for Systematic Reviews and Meta-Analysis Protocols (PRISMA-P) [40], and the populated version is available in Additional file 1 . The systematic review protocol is submitted for registration in the international prospective register of systematic reviews (PROPSERO) and is awaiting a registration number.

\section{Case definitions}

All case definitions for degenerative LSS, knee OA, and hip OA will be included in this study. This includes imaging and clinical diagnoses, as well as combinations of imaging and clinical diagnoses. Imaging diagnoses will be based on radiographic, magnetic resonance imaging, or computerized tomography descriptions of narrowing of the central, or lateral canals of the lumbar spine. As no widely accepted gold standard for the clinical diagnosis of LSS exists, this review will include all definitions. Clinical diagnosis will be based on signs and symptoms of LSS, including but not limited to, reduced walking capacity, symptom relief with sitting, and symptom relief with spinal flexion. Moreover, all clinical diagnoses associated with degenerative LSS (neurogenic claudication, radicular type, and mixed types) will be included [41], as they represent the clinical manifestation of central, lateral, and combined central and lateral canal stenosis, respectively.

\section{Study eligibility criteria}

Studies will be included in this systematic review if they meet the following inclusion and exclusion criteria:

\section{Inclusion criteria}

- Study designs including cross-sectional studies, cohort studies, and randomized controlled trials.
- Studies including adults (18 years or older) with LSS and knee and/or hip OA.

- Studies assessing the prevalence of co-occurring LSS and knee and/or hip OA or presenting sufficient data for estimating the prevalence (number of participants with LSS, number of participants with knee and/or hip OA, and total number of participants).

- Full-text papers published in English in peerreviewed journals.

\section{Exclusion criteria}

- Studies including individuals with low back, knee, or hip pain with other origins (e.g., fracture, tumor, inflammatory disease, infection, and lumbar disc herniation).

- Studies including congenital or non-degenerative forms of LSS, without separate data on degenerative LSS.

- Laboratory studies, cadaveric studies, and conference abstracts.

\section{Search strategy}

A comprehensive search for relevant studies was designed in consultation with a health sciences librarian and will be reviewed by a second librarian using the Peer Review of Electronic Search Strategies (PRESS) Checklist $[42,43]$. The following bibliographic databases will be searched with no publication date or language limitation, but only articles in English will be included: MEDLINE, EMBASE, CENTRAL, and CINAHL. Forward citation tracking will be performed in Web of Science. Search term groups will be combined covering the following domains: LSS, knee OA, and hip OA. The search terms used for each domain were developed based on previous Cochrane reviews on LSS [44] and knee [45] and hip OA [46]. We included search terms related to low back pain in the LSS search domain to increase the sensitivity of the search strategy. Additional file 2 presents the search strategy designed for MEDLINE. A pilot search has been performed using the search terminology to ensure its all-inclusiveness and that sufficient original articles exist to perform this review. Automated search updates will be set up in each database to ensure the inclusion of the latest publications in the field.

Reference lists of retrieved articles and reviews will be scrutinized. Scientific abstracts presented from 2018 onwards at the International Forum for Back and Neck Pain Research in Primary Care and Osteoarthritis Research Society International World Congress will be reviewed to identify relevant studies. Content experts will be contacted to identify additional studies not captured in the bibliographic database search. Content experts known to members of the study team will be identified and these experts 
will be asked to identify additional potential content experts. PROSPERO will also be searched for ongoing or recently completed systematic reviews. All studies identified by our search strategy will be retrieved and managed using Endnote X9 (Thomson Reuters, Philadelphia, PA, USA) and Covidence systematic review software (Veritas Health Innovation, Melbourne, Australia).

\section{Study selection}

All retrieved records will be scrutinized in a two-stage screening process by two independent reviewers. Reviewers will first independently screen titles and abstracts according to eligibility criteria, and disagreements will be discussed until consensus or resolved by a third independent reviewer if necessary. Full-text articles of all studies deemed eligible will be retrieved.

In the second stage, the two reviewers will independently screen the full-text articles against the eligibility criteria. Study authors will be contacted for additional information regarding eligibility criteria if necessary. Disagreements will be resolved using consensus meetings or by a third reviewer if consensus cannot be reached. Reasons for excluding full-text studies will be recorded. Absolute agreement and the Kappa coefficient [47] will be calculated for both phases of screening.

\section{Data extraction}

Data from the included studies will be extracted by two authors independently using a standardized data extraction form developed by the authors for this review. Disagreements will be resolved by discussion until consensus or by including a third reviewer. Authors of included studies with missing data will be contacted when additional information is required for extraction. The data extraction form will be tested on ten randomly selected studies from the pilot search and amended accordingly.

Data extraction will include the following:

- First author, publication year, and country.

- Study topic, objectives, and design.

- Time of study, method of data collection, study population, and health care setting.

- Total sample size, participation and response rate, and cohort characteristics (e.g., mean age, age range, sex distribution, ethnicity, and socioeconomic status).

- Case definitions and clinical presentations of LSS.

- Case definitions of knee and/or hip OA.

- Prevalence of LSS and knee and/or hip OA.

- Reports of pain severity and disability levels.

- Information for assessment of methodological quality.

\section{Risk of bias assessment}

Two members of the study team will independently assess the risk of bias of the included studies. Assessment of the risk of bias will be conducted using a modified version of the risk of bias tool for prevalence studies developed by Hoy et al. [48]. Modifications were made to the risk of bias tool for the purpose of this study. All items on the original tool that made specific reference to low back pain were altered to lumbar spinal stenosis and knee and hip osteoarthritis, where applicable. Item 1 was altered from "a close representation of the national population" to "a close representation of the target population" as this review is not concerned with national populations. Item 5 was removed as clinical and imaging information can only be collected directly from participants, and thus irrelevant to the aims of this review. An additional response option "Irrelevant" was added to item 9 for studies that report imaging diagnoses, as imaging findings are not subject to recall limitations. The modifications to items 1, 5, and 9 have been used previously in a prevalence review for LSS [10]. Additionally, item 6 was split into two items, 6a and $\mathrm{b}$, to assess the acceptability of the case definition for (a) lumbar spinal stenosis and (b) knee and hip osteoarthritis. Item 7 was also divided into two items, $7 \mathrm{a}$ and $\mathrm{b}$, to assess the reliability and validity of the measurement instrument for (a) lumbar spinal stenosis and (b) knee and hip osteoarthritis. The modified risk of bias tool is presented in Additional file 3.

The modified risk of bias tool will be used for all included study designs (including randomized controlled trials), as only cross-sectional data from these designs will be used in prevalence estimates. Individual items on the risk of bias tool will be rated as "Yes" for low risk of bias or "No" for high risk of bias or if there is insufficient information in the study to allow judgment of the particular item. An overall risk of bias (high, moderate, or low) for each study will be determined based on the consensus agreement of all raters, taking into consideration the responses to each item on this tool.

\section{Evidence synthesis}

The selection process will be summarized in a PRISMA flowchart. The results of data extraction and assessment of risk of bias will be summarized in tables. Study and participant characteristics will be reported descriptively. The proportion of participants with LSS reporting cooccurring knee and hip OA will be described as prevalence estimates with $95 \%$ confidence intervals. Estimates of the pooled proportions using a random effects model will be calculated, if possible, for the co-occurrence of LSS with knee and hip OA, respectively. Results for prevalence estimates for multimorbid LSS and knee OA will be presented in Fig. 1, while prevalence estimates for multimorbid LSS and hip OA will be presented in a 


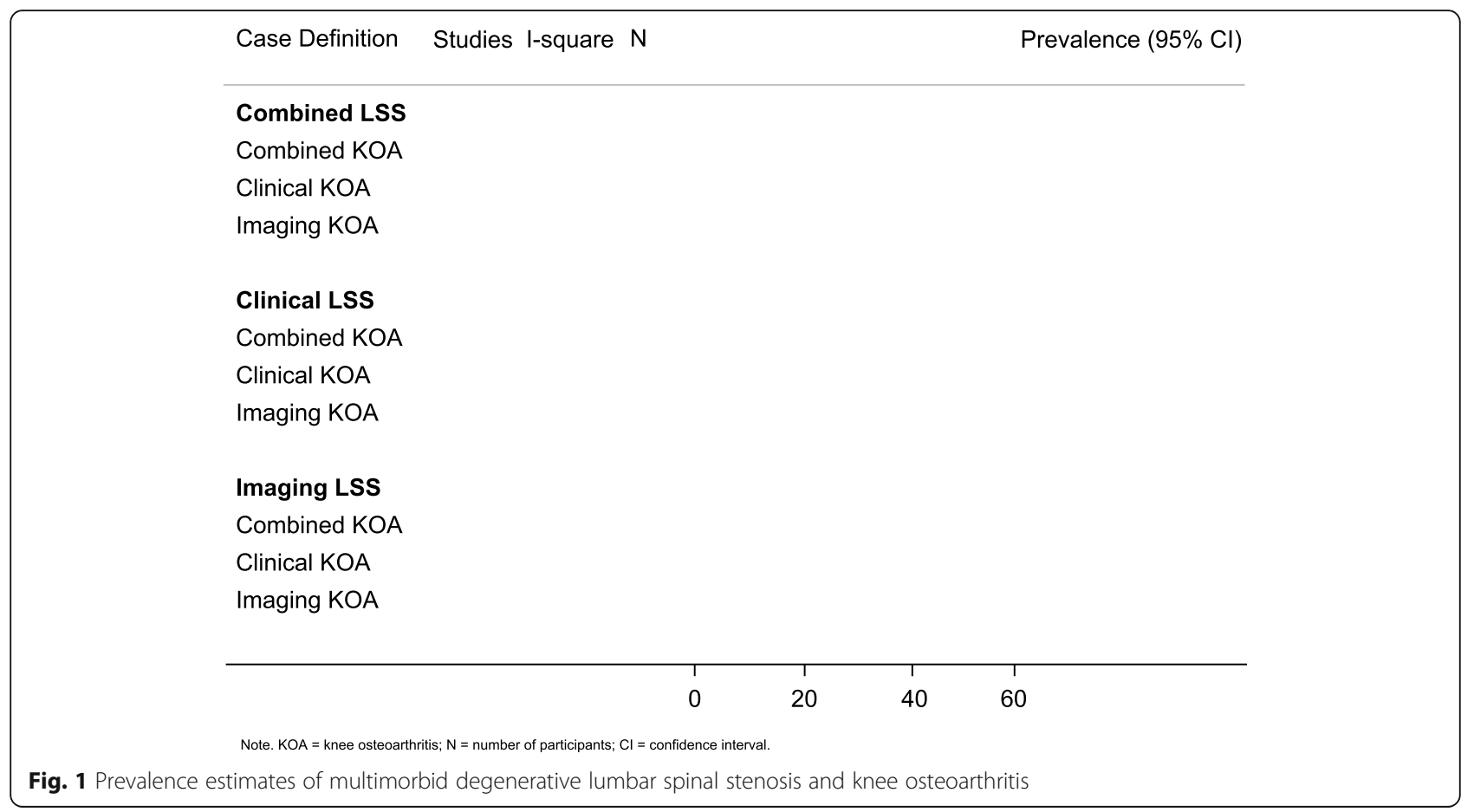

similar figure. If meta-analysis is not possible, a narrative synthesis of included study results will be performed. If it is necessary to perform a narrative synthesis, results will be stratified according to the risk of bias (low, moderate, and high).

Heterogeneity will be evaluated using the $I^{2}$ statistic [39] and Q index [49]. $I^{2}$ values of $0 \%$ represent no inconsistency between the results, and $I^{2}$ values of $100 \%$ represent maximal inconsistency between the results in the included studies. The inconsistency can be considered low if $I^{2}$ is less than 40\%, moderate between 30 and $60 \%$, substantial between 50 and $90 \%$, and considerable between 75 and 100\% [39]. Publication bias will be investigated using a visual examination of the funnel plot. When there is no publication bias, the funnel plot will have a symmetrical, funnel shape, whereas an asymmetrical funnel plot indicates a publication bias [50].

Meta-regression analyses investigating the impact of LSS clinical presentations (neurogenic claudication, radicular type, and mixed type), sample population (general public, occupational), healthcare setting (hospital, community), and risk of bias (low, moderate, and high risk of bias according to the modified risk of bias tool), as well as the covariates age (mean age in the individual studies) and sex (percentage of female participants in the individual studies), if possible. The impact of selfreported LSS pain severity will be evaluated using the visual analog scale for pain and numeric rating scale for pain (computed individually), if sufficient data is available. Self-reported LSS disability will also be investigated individually using the Swiss Spinal Stenosis Questionnaire, and Oswestry Disability Index, or other disability questionnaire scores, when possible. These potential moderators were chosen for investigation as they have been evaluated in previous and ongoing meta-analyses for LSS $[10,51]$. We expect that prevalence is positively associated with increasing age, percentage of female participants, and pain and disability levels. We do not expect prevalence estimates to be impacted by the risk of bias rating of studies or the LSS clinical presentation. A co-variate able to reduce $I^{2}$ (and thus the between study variance tau-square) will be regarded as important for the prevalence of co-occurrence. All statistical analyses will be performed in Stata 16.1 (StataCorp LLC, College Station, USA).

\section{Discussion}

The discussion will include the strengths and limitations of this review. This review is strengthened by the adherence to recommendations from the Cochrane Handbook [39] and reporting according to the PRISMA-P statement [40]. A detailed search strategy, developed in conjunction with a research librarian, performed in multiple databases also strengthens this review. Additionally, the inclusion of all case definitions of LSS and knee/hip OA will provide a comprehensive review of all available literature and the presentation of separate prevalence estimates by case definition for both LSS and knee/hip OA will increase the clinical value. 
This review is not without limitations. The lack of consensus gold standard case definitions for both LSS and OA may limit our ability to draw firm conclusions about the multimorbid prevalence of these conditions. It is likely that clinical diagnoses of LSS and OA are of greater importance than imaging diagnoses due to their relationship with functional impact. However, we have chosen to include all case definitions in this review as we expect there will be limited studies available to estimate the multimorbid prevalence, and we will attempt to provide prevalence estimates for each combination of case definition.

We have chosen to operationalize multimorbidity by simply counting the presence of these conditions, when many other multimorbidity frameworks have been proposed [52] and differing definitions can influence multimorbidity prevalence estimates [53]. It may be that other factors related to multimorbid LSS and OA, such as disability level, health-related quality of life, and others, are of greater importance than the simple presence of these conditions. As this is the first review attempting to estimate the prevalence of multimorbid LSS and OA, the simplest operationalization of multimorbidity was selected. While a more nuanced multimorbidity definition would be ideal, we do not believe sufficient literature exists to perform a review of this nature. This review should be viewed as a starting point aimed at highlighting the potential problem of multimorbid LSS and hip and knee OA, which can be used in the design of more elaborate multimorbidity studies. As such, we have included only English language studies for this review, as we do not expect a large language bias in this research area. However, it is possible relevant studies will not be identified, but it is unlikely the exclusion of a small number of studies will profoundly impact the results of this review.

This review will provide preliminary prevalence estimates of multimorbid LSS and knee and/or hip OA. The results of this review should raise awareness among researchers and clinicians regarding how commonly these conditions coexist and the need to consider multimorbid presentations when assessing and treating patients and evaluating interventions. The generated prevalence estimates should serve as a starting point from which further research can be conducted to better understand the relationship between co-occurring LSS and knee and/or hip OA. The findings will help determine the need for more rigorous epidemiological studies, as well as inform diagnostic and interventional studies for this patient population. It is our hope that the results of this systematic review and meta-analysis will help policymakers better understand the magnitude of this growing healthcare burden, while also helping clinicians and patients access care pathways better suited to manage these complex multimorbid presentations.

\section{Supplementary information}

Supplementary information accompanies this paper at https://doi.org/10. 1186/s13643-020-01478-4.

Additional file 1. PRISMA-P Checklist.

Additional file 2. MEDLINE Search Strategy.

Additional file 3. Risk of Bias Tool.

\section{Abbreviations}

LSS: Lumbar spinal stenosis; OA: Osteoarthritis; PRESS: Peer Review of Electronic Search Strategies Checklist; PRISMA-P: Preferred Reporting Items for Systematic Reviews and Meta-Analysis Protocols

\section{Acknowledgements}

Not applicable.

Ethical approval and consent to participate

Not applicable.

\section{Authors' contributions}

$J J Y, J H, R K J, E R$, and CA conceived the idea, and JJY wrote the protocol with critical revisions from all authors. JJY and CBJ developed the search strategy with assistance from a research librarian. JJY will carry out searches with CBJ, and JJY and co-authors will independently assess studies for inclusion. Data extraction will be done by JJY and co-authors independently. All authors will be involved in the analysis and assessment of methodological quality. JJY will lead the analysis with participation of CBJ. All authors will participate in the interpretation and formation of conclusions. JJY will draft the report with critical revision and contribution from all authors. All authors will give their approval prior to submission for publication of the final version of this systematic review article.

\section{Funding}

JJY has received PhD funding support from the Danish Foundation for Chiropractic Research and Post-graduate Education, the Ontario Chiropractic Association, the Canadian Memorial Chiropractic College, the National Chiropractic Mutual Insurance Company Foundation, and a faculty scholarship from the University of Southern Denmark. No funding body has any role in the conduct of this study.

\section{Availability of data and materials}

The datasets used and/or analyzed during the current study are available from the corresponding author on reasonable request.

Consent for publication

Not applicable.

Competing interests

The authors declare that they have no competing interests.

\section{Author details}

${ }^{1}$ Center for Muscle and Joint Health, University of Southern Denmark, Campusvej 55, 5230 Odense M, Denmark. ${ }^{2}$ Research Division, Canadian Memorial Chiropractic College, 6100 Leslie Street, Toronto, Canada. ${ }^{3}$ Nordic Institute of Chiropractic and Clinical Biomechanics, Odense M, Denmark.

${ }^{4}$ Rebecca MacDonald Centre for Arthritis and Autoimmune Diseases, Mount Sinai Hospital, Toronto, Canada. ${ }^{5}$ Institute for Health Policy, Management and Evaluation, University of Toronto, Toronto, Canada. ${ }^{6}$ Department of Physiotherapy and Occupational Therapy, Copenhagen University Hospital, and Gentofte, Herlev, Denmark.

Received: 1 April 2020 Accepted: 9 September 2020

Published online: 07 October 2020

\section{References}

1. Briggs AM, de Carvalho IA. Actions required to implement integrated care for older people in the community using the World Health Organization's ICOPE approach: a global Delphi consensus study. PloS One. 2018:13(10): e0205533. 
2. Hay SI, Abajobir AA, Abate KH, Abbafati C, Abbas KM, Abd-Allah F, et al. Global, regional, and national disability-adjusted life-years (DALYs) for 333 diseases and injuries and healthy life expectancy (HALE) for 195 countries and territories, 1990-2016: a systematic analysis for the Global Burden of Disease Study 2016. Lancet. 2017;390(10100):1260-344.

3. James SL, Abate D, Abate KH, Abay SM, Abbafati C, Abbasi N, et al. Global, regional, and national incidence, prevalence, and years lived with disability for 354 diseases and injuries for 195 countries and territories, 1990-2017: a systematic analysis for the Global Burden of Disease Study 2017. Lancet. 2018;392(10159):1789-858.

4. Vos T, Abajobir AA, Abate KH, Abbafati C, Abbas KM, Abd-Allah F, et al. Global, regional, and national incidence, prevalence, and years lived with disability for 328 diseases and injuries for 195 countries, 1990-2016: a systematic analysis for the Global Burden of Disease Study 2016. Lancet. 2017;390(10100):1211-59.

5. Jindai $K$, Nielson CM, Vorderstrasse BA, Quiñones AR. Peer reviewed: multimorbidity and functional limitations among adults 65 or older, NHAN ES 2005-2012. Prev Chronic Dis. 2016;13.

6. Lurie J, Tomkins-Lane C. Management of lumbar spinal stenosis. BMJ. 2016; 352:h6234.

7. Fanuele JC, Birkmeyer NJ, Abdu WA, Tosteson TD, Weinstein JN. The impact of spinal problems on the health status of patients: have we underestimated the effect? Spine. 2000;25(12):1509-14.

8. Ferreira ML, De Luca K. Spinal pain and its impact on older people. Best Pract Res Cl Rh. 2017;31(2):192-202.

9. Deyo RA. Treatment of lumbar spinal stenosis: a balancing act. Spine J. 2010;10(7):625-7.

10. Jensen R, Jensen T, Koes B, Hartvigsen J. Prevalence of lumbar spina stenosis in general and clinical populations: a systematic review and metaanalysis. Eur Spine J. 2020.

11. Kalichman L, Cole R, Kim DH, Li L, Suri P, Guermazi A, et al. Spinal stenosis prevalence and association with symptoms: the Framingham Study. Spine J. 2009;9(7):545-50

12. World Health Organization. World report on ageing and health: World Health Organization; 2015: Available from: www.who.int/ageing/events/ world-report-2015-launch/en/.

13. Fritsch CG, Ferreira ML, Maher CG, Herbert RD, Pinto RZ, Koes B, et al. The clinical course of pain and disability following surgery for spinal stenosis: a systematic review and meta-analysis of cohort studies. Eur Spine J. 2017; 26(2):324-35.

14. Jönsson B, Annertz M, Sjöberg C, Strömqvist B. A prospective and consecutive study of surgically treated lumbar spinal stenosis: part II: fiveyear follow-up by an independent observer. Spine. 1997;22(24):2938-44.

15. National Institute for Health and Care Excellence. Multimorbidity: clinical assessment and management. National Institute for Health and Care Excellence Guidelines. 2016;NG56.

16. United States Department of Health and Human Services, Office of Disease Prevention and Health Promotion. Healthy people 2020. United States Department of Health and Human Services. 2011: Available from www. healthypeople.gov/.

17. Smith SM, Wallace E, O'Dowd T, Fortin M. Interventions for improving outcomes in patients with multimorbidity in primary care and community settings. Cochrane Database Syst Rev. 2016;3.

18. Coggon D, Ntani G, Palmer KT, Felli VE, Harari R, Barrero LH, et al. Patterns of multisite pain and associations with risk factors. Pain. 2013:154(9):1769-77.

19. Hartvigsen J, Davidsen M, Hestbaek L, Sogaard K, Roos EM. Patterns of musculoskeletal pain in the population: a latent class analysis using a nationally representative interviewer-based survey of 4817 D anes. Eur J Pain. 2013;17(3):452-60.

20. Kamaleri Y, Natvig B, Ihlebaek CM, Benth JS, Bruusgaard D. Number of pain sites is associated with demographic, lifestyle, and health-related factors in the general population. Eur J Pain. 2008;12(6):742-8.

21. de Luca K, Wong A, Eklund A, Fernandez M, Byles JE, Parkinson L, et al. Multisite joint pain in older Australian women is associated with poorer psychosocial health and greater medication use. Chiropr Man Ther. 2019; 27(1):8.

22. Bastick AN, Runhaar J, Belo JN, Bierma-Zeinstra SM. Prognostic factors for progression of clinical osteoarthritis of the knee: a systematic review of observational studies. Arthritis Res Ther. 2015;17(1):152.

23. Bierma-Zeinstra S, Waarsing J. The role of atherosclerosis in osteoarthritis Best Prac Res CI Rh. 2017;31(5):613-33.
24. Calders P, Van Ginckel A. editors. Presence of comorbidities and prognosis of clinical symptoms in knee and/or hip osteoarthritis: a systematic review and meta-analysis. Semin Arthritis Rheum. 2018;47(6):805-13.

25. Wolfe F, Hawley DJ, Peloso PM, Wilson K, Anderson J. Back pain in osteoarthritis of the knee. Arthritis Rheum. 1996;9(5):376-83.

26. Dutton R. A Review of Hip-Spine Syndrome. Curr Phys Med Rehabil Rep. 2019;7(3):264-74.

27. Prather $H$, van Dillen L. A narrative review of the links between the hip and the lumbar spine (hip spine syndrome) as they relate to clinical decision making for patients with lumbopelvic pain. PM \& R. 2019;11:s64-72.

28. Ferrero E, Lonjon G, Bouyer B, Sabourin M, Ould-Slimane M, Guigui P. Influence of comorbidities on patients reported outcomes in degenerative lumbar spinal stenosis. Orthop Traumatol Surg Res. 2018;104(7):1031-6.

29. Offierski C, MacNab I. Hip-spine syndrome. Spine. 1983;8(3):316-21.

30. Ishimoto $Y$, Yoshimura N, Muraki S, Yamada H, Nagata K, Hashizume $H$, et al. Associations between radiographic lumbar spinal stenosis and clinical symptoms in the general population: the Wakayama Spine Study. Osteoarthritis Cartilage. 2013;21(6):783-8.

31. Jordan JM, Helmick CG, Renner JB, Luta G, Dragomir AD, Woodard J, et al. Prevalence of hip symptoms and radiographic and symptomatic hip osteoarthritis in African Americans and Caucasians: the Johnston County Osteoarthritis Project. J Rheumatol. 2009;36(4):809-15.

32. Kim C, Nevitt MC, Niu J, Clancy MM, Lane NE, Link TM, et al. Association of hip pain with radiographic evidence of hip osteoarthritis: diagnostic test study. BMJ. 2015;351:h5983.

33. Devin CJ, McCullough KA, Morris BJ, Yates AJ, Kang JD. Hip-spine syndrome. J Am Acad Orthop Surg. 2012;20(7):434-42.

34. Cross M, Smith E, Hoy D, Nolte S, Ackerman I, Fransen M, et al. The global burden of hip and knee osteoarthritis: estimates from the global burden of disease 2010 study. Ann Rheum Dis. 2014;73(7):1323-30.

35. Ito H, Tominari S, Tabara Y, Nakayama T, Furu M, Kawata T, et al. Low back pain precedes the development of new knee pain in the elderly population; a novel predictive score from a longitudinal cohort study. Arthritis Res Ther. 2019;21(1):98.

36. Goode AP, Cleveland RJ, George SZ, Kraus VB, Schwartz TA, Gracely RH, et al. Different phenotypes of osteoarthritis in the lumbar spine reflected by demographic and clinical characteristics: the Johnston County Osteoarthritis Project. Arthritis Care Res. 2020;72(7):974-81.

37. Wang L, Si L, Cocker F, Palmer AJ, Sanderson K. A systematic review of cost-ofillness studies of multimorbidity. Appl Health Econ Hea Pol. 2018;16(1):15-29.

38. Pearson-Stuttard J, Ezzati M, Gregg EW. Multimorbidity - a defining challenge for health systems. Lancet Public Health. 2019;4(12):e599-600.

39. Higgins JP, Thomas J, Chandler J, Cumpston M, Li T, Page MJ, et al. Cochrane Handbook for Systematic Reviews of Interventions version 6.0 (updated July 2019). The Cochrane Collaboration. 2019: Available from www.training.cochrane.org/handbook.

40. Shamseer L, Moher D, Clarke M, Ghersi D, Liberati A, Petticrew M, et al. Preferred reporting items for systematic review and meta-analysis protocols (PRISMA-P) 2015: elaboration and explanation. BMJ. 2015;349:g7647.

41. Suri P, Rainville J, Kalichman L, Katz JN. Does this older adult with lower extremity pain have the clinical syndrome of lumbar spinal stenosis? JAMA. 2010;304(23):2628-36.

42. McGowan J, Sampson M, Lefebvre C. An evidence based checklist for the peer review of electronic search strategies (PRESS EBC). Evid Based Libr Inf Pract. 2010:5(1):149-54

43. McGowan J, Sampson M, Salzwedel DM, Cogo E, Foerster V, Lefebvre C. PRESS peer review of electronic search strategies: 2015 guideline statement J Clin Epidemiol. 2016;75:40-6.

44. Ammendolia C, Stuber KJ, Rok E, Rampersaud R, Kennedy CA, Pennick V, et al. Nonoperative treatment for lumbar spinal stenosis with neurogenic claudication. Cochrane Database Syst Rev. 2013;8:CD010712.

45. Fransen M, McConnell S, Harmer AR, Van der Esch M, Simic M, Bennell KL. Exercise for osteoarthritis of the knee. Cochrane Database Syst Rev. 2015;1: CD004376.

46. Fransen M, McConnell S, Hernandez-Molina G, Reichenbach S. Exercise for osteoarthritis of the hip. Cochrane Database Syst Rev. 2014:4:CD007912.

47. Cohen J. A coefficient of agreement for nominal scales. Educ Psychol Meas. 1960;20:37-46

48. Hoy D, Brooks P, Woolf A, Blyth F, March L, Bain C, et al. Assessing risk of bias in prevalence studies: modification of an existing tool and evidence of interrater agreement. J Clin Epidemiol. 2012;65(9):934-9. 
49. Higgins JP, Thompson SG, Deeks JJ, Altman DG. Measuring inconsistency in meta-analyses. BMJ. 2003;327(7414):557-60.

50. Sterne JA, Egger M. Funnel plots for detecting bias in meta-analysis: guidelines on choice of axis. J Clin Epidemiol. 2001;54(10):1046-55.

51. Ferretti F, Coluccia A, Gusinu R, Gualtieri G, Muzii VF, Pozza A. Quality of life and objective functional impairment in lumbar spinal stenosis: a protocol for a systematic review and meta-analysis of moderators. BMJ Open. 2019;9(11):e032314.

52. Willadsen $T G$, Bebe A, Køster-Rasmussen R, Jarbøl DE, Guassora AD, Waldorff FB, et al. The role of diseases, risk factors and symptoms in the definition of multimorbidity-a systematic review. Scand J Prim Health Care. 2016;34(2):112-21.

53. Griffith LE, Gilsing A, Mangin D, Patterson C, van den Heuvel E, Sohel N, et al. Multimorbidity frameworks impact prevalence and relationships with patient-important outcomes. J Am Geriatr Soc. 2019;67(8):1632-40.

\section{Publisher's Note}

Springer Nature remains neutral with regard to jurisdictional claims in published maps and institutional affiliations.

Ready to submit your research? Choose BMC and benefit from:

- fast, convenient online submission

- thorough peer review by experienced researchers in your field

- rapid publication on acceptance

- support for research data, including large and complex data types

- gold Open Access which fosters wider collaboration and increased citations

- maximum visibility for your research: over $100 \mathrm{M}$ website views per year

At BMC, research is always in progress.

Learn more biomedcentral.com/submissions 